\title{
Endophenotypic Effects of the SORL1 Variant rs2298813 on Regional Brain Volume in Patients with Alzheimer's Disease
}

\section{Chun-Yu Chen}

Taipei Veterans General Hospital

\section{Yung-Shuan Lin}

Taipei Veterans General Hospital

Wei-Ju Lee

Taichung Veterans General Hospital

Yi-Chu Liao

Taipei Veterans General Hospital

\section{Yu-Shan Kuo}

Taipei Veterans General Hospital

Albert C Yang

Taipei Veterans General Hospital

Jong-Ling Fuh ( $\nabla$ jlfuh@vghtpe.gov.tw)

Taipei Veterans General Hospital

\section{Research Article}

Keywords: Alzheimer's disease, sortilin-related receptor 1 gene, MRI

Posted Date: February 15th, 2022

DOI: https://doi.org/10.21203/rs.3.rs-1348025/v1

License: (9) (i) This work is licensed under a Creative Commons Attribution 4.0 International License. Read Full License 


\section{Abstract}

\section{Background}

Two common variants of sortilin-related receptor 1 gene (SORL 1), rs2298813 and rs1784933, have been associated with late-onset Alzheimer's disease (AD) in the Han Chinese population in Taiwan. However, neuroimaging correlates of these two SORL 1 variants remain unknown. We aimed to determine whether the two SORL 1 polymorphisms were associated with any volumetric differences in brain regions in AD patients.

\section{Methods}

We recruited 200 patients with late-onset AD from Taipei Veterans General Hospital. All patients received a structural magnetic resonance imaging brain scan and completed a battery of neurocognitive tests at enrollment. We followed up to assess changes in Mini-Mental State Examination (MMSE) scores in 155 patients $(77.5 \%)$ at an interval of 2 years. Volumetric measures and cortical thickness of various brain regions were performed using FreeSurfer. Regression analysis controlled for apolipoprotein E status. Multiple comparisons were corrected for using the false discovery rate.

\section{Results}

The rs2298813 and rs1783933 genotypes were not associated with cognitive test performance or clinical progression. The homozygous major allele of rs2298813 was associated with larger volumes in the right putamen $(p=0.0442)$ and right pallidum $(p=0.0346)$. There was no link of the rs 1784933 genotypes with any of the imaging measures. In the rs2298813 homozygous major allele carriers, the right putaminal volume was associated with verbal fluency $(p=0.008)$ and clinical progression $(p=0.020)$. In the minor allele carriers, the right putaminal and right pallidal volumes were not significantly related to cognitive test scores or clinical progression.

\section{Conclusions}

The major and minor alleles of rs2298813 had differential effects on the right lentiform nucleus volume and distinctively modulated the association between the regional volume and cognitive function in patients with Alzheimer's disease.

\section{Background}

Accumulation of the neurotoxic proteolytic derivative of amyloid beta precursor protein (APP), amyloidbeta $(A B)$ peptide, is proposed to be key to the pathogenesis of Alzheimer's disease (AD) [1]. In support of this hypothesis, associations between early-onset familial AD and mutations in APP provide the strongest genetic evidence, with additional evidence from mutations in PSEN1 and PSEN2 [2]. With respect to late-onset $A D$, the apolipoprotein $E$ gene $(A P O E)$ is the major genetic risk factor, and a wide diversity of common variants have been identified in a number of genes, e.g., BIN1, CR1, ABCA7, PICALM, 
CD33, EPHA1, and CLU[3]. The sortilin-related receptor 1 gene (SORL1), encoding a sorting-related receptor with type-A repeats (SORLA) involved in intracellular trafficking and processing of APP, is an important gene among these [4].

SORL 1 variants were first identified, among several genes belonging to endocytic pathways, to be associated with sporadic AD by a pioneering study in Caucasians [5]. Targeted SNP analyses and genome-wide association studies have validated the association not only in populations of Caucasian origin but also in Asian populations [6-8]. It has been revealed that SORL 1 expression is reduced in the brains of $A D$ patients [9], and ablation of SORLA expression increases $A \beta$ in the brains of knockout mice [10]. A number of studies have shown significant associations between specific SORL 1 polymorphisms and lower $A \beta$ levels in cerebrospinal fluid [11] and serum [12]. Furthermore, previous studies have demonstrated that various SORL 1 polymorphisms are associated with hippocampal atrophy in $A D$ patients [13].

We have previously reported that in the Han Chinese population in Taiwan, two common variants of SORL 1, rs2298813 and rs1784933, were associated with late-onset AD [12]. Rs2298813 is located at the vacuolar protein sorting 10 protein (VPS10P) domain, and the nonsynonymous substitution of alanine to threonine at the 528th residue (A528T) of SORLA increased the secretion of Aß42 [14]. Rs1784933 is located in the $3^{\prime}$ region of $S O R L 1$, and minor allele carriers had lower plasma concentrations of $A \beta 42$ [12]. The endophenotypic effects of various SORL 1 polymorphisms on the brain have been revealed in nondemented individuals [15-18]. However, the neuroimaging correlates of the two SORL 1 variants have not been established in AD patients. We therefore aimed to determine whether the two SORL1 polymorphisms rs2298813 and rs1784933 are associated with differences in AD-related brain regions in $A D$ patients and to further determine whether there are any neuroimaging correlates beyond AD-related brain regions.

\section{Methods}

\section{Subjects}

A total of 200 patients with late-onset AD were enrolled from Taipei Veterans General Hospital, Taiwan. All participants were of Han Chinese descent in Taiwan. Probable AD was diagnosed based on the criteria of the National Institute of Neurological and Communicative Disorders and Stroke/Alzheimer's Disease and Related Disorders Association [19]. The diagnostic survey included history queries, neurological examinations, laboratory tests, and magnetic resonance (MR) imaging of the brain. A subset of patients ( $n=92)$ were screened for cognitive fluctuation using the Mayo fluctuation scale [20]. The study was approved by the institutional review boards of Taipei Veterans General Hospital. Informed consent was obtained from all patients in accordance with our institutional guidelines and the recommendations of the Declaration of Helsinki.

\section{Genotyping}


Whole blood genomic DNA was extracted with a commercial kit in accordance with the manufacturer's instructions (QIAGEN, Hilden, Germany). The alleles of $\operatorname{APOE}(\varepsilon 2, \varepsilon 3$, and $\varepsilon 4)$ were determined by rs429358 and rs7412 [21]. Genotyping of the two SORL1 SNPs (rs2298813 and rs1784933) and APOE alleles was performed using the TaqMan genotyping assay (Applied Biosystems, Foster City, CA, USA). Polymerase chain reactions were carried out in 96-well microplates using an ABI 7500 real-time polymerase chain reaction system (Applied Biosystems International, Framingham, MA). For allele discrimination, the fluorescence signal from the TaqMan polymerase chain reaction was analyzed using SDS software version 1.2.3 (Applied Biosystems International, Framingham, MA).

\section{Cognitive testing}

Global cognitive performance was assessed in each patient using the Mini-Mental State Examination (MMSE) [22]. Cognitive domain-specific tests were performed in all patients. Attention was tested by the forward and backward digit span tests from the Wechsler Memory Scale-IV [23], memory by the 12-item word recall test [24], language and executive function by the verbal fluency category test [25], processing speed by the Trail Making Test A [26], and naming by the Boston naming test [27]. We followed up to assess changes in MMSE scores over a mean interval of approximately 2 years in these patients. Rapid clinical progression was defined as a decrease in follow-up MMSE by at least 3 points per year [28].

\section{Imaging analysis}

MR images were scanned at Taipei Veterans General Hospital, Taipei, Taiwan, on a 3.0-T GE Signa MRI scanner (GE Medical Systems, Milwaukee, WI, USA). High-resolution anatomic MR images were acquired through a 3D inversion-recovery fast spoiled gradient-echo (BRAVO) sequence. The high-resolution structural T1 images were processed using FreeSurfer version 5.3 (http://surfer.nmr.mgh.harvard.edu/) based on the 2010 Desikan-Killiany atlas. Cortical reconstruction using FreeSurfer involved automated and manual processing. The automated processing included motion correction, nonbrain tissue removal [29], Talairach transformation, segmentation of the subcortical white matter and deep gray matter structures, intensity normalization, tessellation of the boundary between gray and white matter [30], automated topology correction, and surface deformation. When necessary, manual editing was undertaken to correct the pial surface error, skull strip error, or intensity normalization error following the FreeSurfer tutorial. Cortical thickness was calculated as the distance between the white and gray matter surfaces at each point across the regional cortex. There were a total of 68 regions of cortical thickness and 21 regions of gray matter volume included in the statistical analysis. AD-related brain regions included volumes in the hippocampus and thickness of the parahippocampal gyrus, posterior cingulate, middle temporal gyrus, and entorhinal cortex [17]. Other regions (other than AD-related regions) included all regions except the AD-related brain regions (19 regions of gray matter volume and 60 regions of cortical thickness).

\section{Statistical analysis}


Hardy-Weinberg equilibrium tests were conducted for each SNP. A dominant model of inheritance of the minor allele was presumed to test the associations between SORL 1 SNPs and imaging parameters. The analyses were executed with PASW Statistics software (version 25.0; SPSS, Chicago, IL, USA).

Data are expressed as the mean \pm SD or number of patients (\%), as appropriate. The $\chi 2$ test was performed for categorical variables, and the $t$ test was performed for comparison of two means. Multivariate linear regression analyses were used to assess the relationships between regional cortical thickness or gray matter volume and the SORL 1 SNPS or cognitive test results. The covariates included age, sex, education level, and APOE status. A logistic regression model was conducted to investigate the associations between rapid clinical progression or MMSE changes at follow-up and features of selected brain regions with covariates that included age, sex, education level, and APOE status. For regression analysis involving gray matter volume, the estimated intracranial volume was additionally included as a covariate. Multiple comparisons were corrected with the false discovery rate (Benjamini-Hochberg procedure) respectively for gray matter volume and cortical thickness. Statistical significance was taken at $\mathrm{P}<0.05$ or Benjamini-Hochberg corrected $\mathrm{P}_{\mathrm{C}}<0.05$.

\section{Results}

\section{Demographic data}

The demographic data for those with rs2298813 and rs1784933 are shown in Table 1. Regarding rs2298813, there were 147 patients carrying the wild homozygote (GG), and 51 minor allele carriers (51 $A G$ and $3 A A)$. Regarding rs 1784933, there were 92 patients carrying the wild homozygote (AA), and 109 minor allele carriers (93 AG and $16 \mathrm{GG}$ ). Age, sex, MMSE score, years of education, disease duration, and APOE status did not differ between the homozygous major allele carriers and the minor allele carriers of either rs2298813 or rs1784933. The Mayo fluctuation scale scores tended to be higher in minor allele carriers of rs 2298813 than in homozygous major allele carriers $(p=0.052)$ but were similar between minor allele carriers and noncarriers of rs1784933 ( $p=0.935)$. For both rs2298813 and rs 1784933 , the minor allele carriers performed similarly in all the neuropsychiatric tests as did the homozygous major allele carriers (Table 1). A total of 155 patients (77.5\%) had a follow-up MMSE assessment after a mean interval of $2.1 \pm 0.8$ years, and 37 patients had clinical progression. At follow-up, the minor allele carriers of rs2298813 or rs1784933 showed no differences in the risk of clinical progression versus the homozygous major allele carriers.

Table 1 Demographic data and cognitive tests of the minor allele carriers and non-carriers 


\begin{tabular}{|c|c|c|c|c|}
\hline \multicolumn{2}{|c|}{ Rs2298813 } & $\mathrm{GG}(\mathrm{n}=146)$ & $A G(n=51) / A A(n=3)$ & $P$ \\
\hline \multicolumn{2}{|c|}{ Age, years } & $77.5 \pm 7.9$ & $78.2 \pm 6.7$ & 0.590 \\
\hline \multicolumn{2}{|c|}{ Male sex } & $67(45.9 \%)$ & $23(42.6 \%)$ & 0.677 \\
\hline \multicolumn{2}{|c|}{ MMSE score } & $19.9 \pm 5.1$ & $20.9 \pm 5.2$ & 0.197 \\
\hline \multicolumn{2}{|c|}{ 12-item word recall } & $1.5 \pm 2.0$ & $2.1 \pm 2.5$ & 0.127 \\
\hline \multicolumn{2}{|c|}{ Forward digit span } & $8.7 \pm 2.9$ & $8.8 \pm 2.7$ & 0.900 \\
\hline \multicolumn{2}{|c|}{ Backward digit span } & $4.2 \pm 1.8$ & $4.6 \pm 2.4$ & 0.290 \\
\hline \multicolumn{2}{|c|}{ Verbal fluency } & $7.1 \pm 2.9$ & $7.9 \pm 2.9$ & 0.079 \\
\hline \multicolumn{2}{|c|}{ Boston Naming } & $11.1 \pm 2.5$ & $11.2 \pm 2.6$ & 0.808 \\
\hline \multicolumn{2}{|c|}{ Trail Making, seconds } & $146.4 \pm 97.4$ & $128.9 \pm 81.0$ & 0.261 \\
\hline \multicolumn{2}{|c|}{ Education level, years } & $10.0 \pm 4.2$ & $9.1 \pm 5.0$ & 0.240 \\
\hline \multicolumn{2}{|c|}{ Disease duration, months } & $31.8 \pm 42.5$ & $32.9 \pm 29.4$ & 0.860 \\
\hline \multirow[t]{2}{*}{ APOE4 } & $\nabla 4$ & $41(28.1 \%)$ & $24(44.4 \%)$ & \multirow[t]{2}{*}{0.074} \\
\hline & $\llbracket 4 / \varangle 4$ & $4(2.7 \%)$ & $2(3.7 \%)$ & \\
\hline \multicolumn{2}{|c|}{ Mayo fluctuations scale } & $1.2 \pm 1.3$ & $1.8 \pm 1.1$ & 0.052 \\
\hline \multicolumn{2}{|c|}{ Clinical progression } & $26 / 118(22.0 \%)$ & $10 / 38(26.3)$ & 0.586 \\
\hline \multicolumn{2}{|c|}{ Rs1784933 } & $A A(n=92)$ & $A G(n=92) / G G(n=16)$ & $\mathrm{P}$ \\
\hline \multicolumn{2}{|c|}{ Age (years) } & $77.7 \pm 8.2$ & $77.55 \pm 7.38$ & 0.870 \\
\hline \multicolumn{2}{|c|}{ Gender (male) } & $45(48.9 \%)$ & $45(41.7 \%)$ & 0.305 \\
\hline \multicolumn{2}{|l|}{ MMSE } & $19.8 \pm 5.4$ & $20.5 \pm 4.7$ & 0.313 \\
\hline \multicolumn{2}{|c|}{ 12-item word recall } & $1.7 \pm 2.1$ & $1.7 \pm 2.3$ & 0.983 \\
\hline \multicolumn{2}{|c|}{ Forward digit span } & $8.5 \pm 2.9$ & $8.9 \pm 2.8$ & 0.253 \\
\hline \multicolumn{2}{|c|}{ Backward digit span } & $4.2 \pm 1.9$ & $4.4 \pm 2.0$ & 0.415 \\
\hline \multicolumn{2}{|c|}{ Verbal fluency } & $7.2 \pm 3.1$ & $7.4 \pm 2.8$ & 0.696 \\
\hline \multicolumn{2}{|c|}{ Boston Naming } & $11.0 \pm 2.7$ & $11.3 \pm 2.4$ & 0.367 \\
\hline \multicolumn{2}{|c|}{ Trail Making, seconds } & $145.7 \pm 96.1$ & $140.7 \pm 93.7$ & 0.715 \\
\hline \multicolumn{2}{|c|}{ Education (years) } & $9.4 \pm 4.2$ & $10.0 \pm 4.5$ & 0.341 \\
\hline \multicolumn{2}{|c|}{ Disease duration (months) } & $27.6 \pm 25.0$ & $36.1 \pm 48.0$ & 0.111 \\
\hline
\end{tabular}




\begin{tabular}{lllll} 
APOE4 & \multicolumn{1}{c}{$\varangle 4$} & $29(31.5 \%)$ & $36(33.3 \%)$ & 0.770 \\
\cline { 1 - 2 } & $2(2.2 \%)$ & $4(3.7 \%)$ & \\
\hline Mayo fluctuations scale & $1.4 \pm 1.3$ & $1.4 \pm 1.2$ & 0.935 \\
\hline Clinical progression & $19 / 67(28.4 \%)$ & $18 / 88(20.5 \%)$ & 0.253
\end{tabular}

APOE, apolipoprotein E; MMSE, Mini-Mental State Examination.

\section{Associations of SORL1 SNPs with regional cortical thickness and gray matter volume}

The correlations between the SORL 1 common variants and regional gray matter volumes or regional cortical thickness in AD-related brain regions and other brain regions are shown in Tables 2 and 3 , respectively. The genotype of rs2298813 was not associated with any of the AD-related brain regions (Table 2). Among other brain regions, there were significant partial correlations of volumes in the right putamen and right pallidum with the genotype of rs2298813; the homozygous major allele was associated with larger volumes in the two regions (Figure 1, Table 3). With respect to rs1784933, there was no association with any of the brain regions.

Table 2 Correlations of regional cortical thickness/gray matter volume with homozygous major alleles of rs2298813 and rs1784933 in Alzheimer disease (AD)-related brain regions

\begin{tabular}{llllllll} 
& \multicolumn{1}{l}{ Rs2298813 } & \multicolumn{3}{c}{ Rs1784933 } \\
\hline AD-related brain regions & $r$ & $p$ & $p_{c}$ & $r$ & $p$ & $p_{c}$ \\
\hline Gray matter volume & & & & & & \\
\hline Right hippocampus & 0.062 & 0.392 & 0.784 & 0.096 & 0.181 & 0.362 \\
\hline Left hippocampus & 0.056 & 0.441 & 0.441 & 0.069 & 0.338 & 0.676 \\
\hline Cortical thickness & & & & & & \\
\hline Right parahippocampal gyrus & -0.122 & 0.0874 & 0.350 & 0.007 & 0.921 & 0.921 \\
\hline Left parahippocampal gyrus & -0.162 & 0.0231 & 0.185 & 0.040 & 0.574 & 0.765 \\
\hline Right posterior cingulate gyrus & -0.047 & 0.517 & 1.000 & -0.087 & 0.224 & 0.896 \\
\hline Left posterior cingulate gyrus & 0.040 & 0.582 & 0.931 & 0.051 & 0.478 & 0.765 \\
\hline Right middle temporal gyrus & 0.080 & 0.263 & 0.701 & 0.121 & 0.0898 & 0.718 \\
\hline Left middle temporal gyrus & 0.032 & 0.652 & 0.869 & 0.073 & 0.311 & 0.622 \\
\hline Right entorhinal cortex & -0.017 & 0.810 & 0.926 & 0.074 & 0.301 & 0.803 \\
\hline Left entorhinal cortex & -0.016 & 0.820 & 0.820 & 0.030 & 0.681 & 0.778
\end{tabular}




\section{r, partial correlation coefficient}

Table 3 Correlations of regional cortical thickness/gray matter volume with homozygous major alleles of rs2298813 and rs1784933 in other brain regions.

\begin{tabular}{llll} 
Rs2298813 & $\mathrm{p}$ & $\mathrm{p}$ & $\mathrm{p}_{\mathrm{c}}$ \\
\hline Gray matter volume & & & \\
\hline Right putamen & 0.217 & 0.00233 & 0.0442 \\
\hline Right pallidum & 0.207 & 0.00364 & 0.0346 \\
\hline Rs1784933 & $\mathrm{p}$ & $\mathrm{p}$ & $\mathrm{p}_{\mathrm{c}}$ \\
\hline Cortical thickness & & & \\
\hline Right superior temporal gyrus & 0.171 & 0.0163 & 0.978 \\
\hline Right postcentral gyrus & 0.154 & 0.0307 & 0.921 \\
\hline Right pars triangularis & 0.149 & 0.0370 & 0.740 \\
\hline Left precentral gyrus & 0.144 & 0.0444 & 0.666
\end{tabular}

r, partial correlation coefficient

\section{Associations of selected brain regions with cognitive test performance}

The associations between selected brain regions and cognitive test performance for the rs2298813 homozygous major allele carriers and minor allele carriers are shown in Table 4. Among the rs2298813 homozygous major allele carriers, the right putaminal volume was significantly related to verbal fluency $(p=008)$. At follow-up, the volumes in the right putamen and right pallidum were positively related to the annual rate of change in MMSE scores (partial $r=0.181, p=0.056$ for the right putamen; partial $r=0.192$, $p=0.042$ for the right pallidum). Logistic regression revealed that lower volumes in both the right putamen $(p=0.020)$ and the right pallidum $(p=0.013)$ were predictive of clinical progression.

Among the minor allele carriers, the left parahippocampal gyrus was significantly related to MMSE scores $(p=0.008)$ and the Boston naming test scores $(p=0.005)$ and tended to be related to verbal fluency $(p=$ 0.051 ; Table 4). Volumes of the right putamen and the right pallidum were not associated with any of the cognitive test results. At follow-up, neither the right putaminal nor pallidal volumes were associated with the annual rate of change in MMSE scores (partial $r=0.043, p=0.814$ for the right putamen; partial $r=$ $-0.138, p=0.444$ for the right pallidum) or clinical progression $(p=0.791$ for the right putamen, $p=0.191$ for the right pallidum).

Table 4 Correlations of selected brain regions with cognitive tests in homozygous major allele carriers (GG) and minor allele carriers (AG or AA) of rs2298813 


\begin{tabular}{|c|c|c|c|c|c|c|c|c|}
\hline & \multicolumn{4}{|c|}{$\mathrm{GG}(\mathrm{n}=146)$} & \multicolumn{4}{|c|}{$A G(n=51) / A A(n=3)$} \\
\hline & \multicolumn{2}{|c|}{ Right putamen } & \multicolumn{2}{|c|}{ Right pallidum } & \multicolumn{2}{|c|}{ Right putamen } & \multicolumn{2}{|c|}{ Right pallidum } \\
\hline & $r$ & $\mathrm{p}$ & $r$ & $\mathrm{p}$ & $r$ & $\mathrm{p}$ & $r$ & $\mathrm{p}$ \\
\hline MMSE & 0.161 & 0.056 & 0.061 & 0.473 & 0.151 & 0.302 & 0.131 & 0.371 \\
\hline 12-item word recall & 0.088 & 0.299 & 0.020 & 0.815 & -0.149 & 0.311 & -0.093 & 0.529 \\
\hline Forward digit span & 0.121 & 0.152 & -0.030 & 0.723 & 0.239 & 0.098 & 0.223 & 0.123 \\
\hline Backward digit span & 0.013 & 0.878 & 0.016 & 0.847 & 0.098 & 0.501 & 0.186 & 0.201 \\
\hline Verbal fluency & 0.224 & 0.008 & 0.058 & 0.494 & 0.037 & 0.802 & 0.057 & 0.696 \\
\hline Boston Naming & 0.100 & 0.238 & 0.092 & 0.276 & 0.159 & 0.276 & 0.209 & 0.149 \\
\hline Trail Making, seconds & -0.146 & 0.090 & 0.009 & 0.916 & -0.121 & 0.435 & -0.086 & 0.578 \\
\hline
\end{tabular}

MMSE, Mini-Mental State Examination; r, partial correlation coefficient.

\section{Discussion}

We found that the homozygous major allele of rs2298813 was associated with larger volumes in the right putamen and right pallidum. There was an association between verbal fluency performance and right putaminal volume, and the right putaminal and pallidal volumes were predictive of clinical progression. However, the rs2298813 minor allele was associated with a higher cognitive fluctuation score and smaller right putaminal and right pallidal volumes, which were not related to neurocognitive test performance or predictive of clinical progression at follow-up. With respect to rs1784933, there were no neuroimaging correlates of the genotypes. It appeared that different genotypes of rs2298813 had differential effects on the volume of right lentiform nucleus and differentially modulated the association between right lentiform nucleus and cognitive function.

The earliest associations between rs2298813 or rs 1784933 and brain atrophy were delineated in a collection of autopsied AD brains [13]. The 3-SNP haplotypes containing the rs 1784933 region were related to pathological scoring and MRI traits of hippocampal atrophy in white patients, whereas no significant associations were identified for rs2298813 [13]. A study using the Alzheimer's Disease Neuroimaging Initiative database investigated the effect of eight SORL 1 SNPS, including rs2298813 and rs1784933, on AD-related brain atrophy in subjects with normal cognition or mild cognitive impairment (MCl) [17]. The A allele of rs2298813 tended to be associated with a lower volume in the right parahippocampal gyrus, and the $\mathrm{G}$ allele of rs1784933 was found to be associated with a higher rate of atrophy in the right parahippocampal gyrus across a 2-year span [17]. In a young healthy Caucasian population, over 100 SNPs (but not including rs2298813) in and surrounding SORL 1 were examined to determine any association with hippocampal volume [31]. However, rs1784933 was not found to have an association. The discrepancy of the relationship between rs2298813/rs1784933 and 
brain atrophy may lie in ethnic differences. SORL 1 SNPs near the $5^{\prime}$ end are associated with AD in European white, Caribbean Hispanic, and Israeli-Arab subjects [5,8], and AD-related SNPs are closer to the 3 ' end in African American, Han Chinese, and Japanese subjects [12].

The putamen and pallidum (forming the lentiform nucleus) are essential elements of the extrapyramidal system and are usually involved in motor disturbances, such as parkinsonism or Huntington disease [32]. Although not included among the typical AD-related brain structures, numerous studies have disclosed an association of these regions with $A D$. The volume of the putamen has been shown to be reduced in $A D$ brains relative to subjects with $\mathrm{MCl}$ or normal aging [33-38]. Such reductions can occur as early as in the prodromal stage [36]. Atrophy in the pallidum has not been consistently observed in previous studies $[34,38]$. The pallidum is relatively resistant to degeneration even in moderate stages of the disease [33] although mild atrophy may be observed [37,39,40]. Pathological studies have demonstrated heavy deposits of $A D$ pathology in the putamen and less in the pallidum [41]. Similarly, iron detection using MR techniques (quantitative susceptibility mapping or phase imaging) has revealed iron accumulation in the putamen and pallidum [42,43], which was associated with higher amyloid PET standardized uptake value ratios [42]. The association of putaminal or pallidal atrophy with common variants of SORL 1 has not been previously specified in AD patients. The most relevant study was conducted by Huang et al. investigating the effect of rs3824968 on gray matter volume in a nondemented Chinese population across a wide age span [15]. Participants carrying the A allele had accelerated reductions with age in the right putamen volume [15]. Thus, we are the first to identify a link between putaminal/pallidal atrophy associated with SORL 1 polymorphisms in AD.

The putamen and pallidum also have functions related to cognition. In patients with Parkinson's disease, deep brain stimulation of the pallidum has been shown to improve verbal fluency.[44] In human immunodeficiency virus-associated neurocognitive impairment, there was an association between cognitive impairment and putaminal volume [45]. Among patients with behavioral variant frontotemporal dementia, there is a relationship between atrophy in the putamen and theory of mind impairment [46]. In support of this, in our study, relationships between both verbal fluency and annual rate of changes in MMSE scores and right putaminal volume were observed in rs2298813 homozygous major allele carriers. In contrast, there were no significant associations between right putaminal/right pallidal volumes and cognitive test performance or clinical progression in the minor allele carriers, suggesting that in these patients, atrophy in the lentiform nucleus may have an effect beyond cognition.

The link between rs2298813 and the lentiform nucleus volume may provide a pathophysiological basis for the association between AD and parkinsonism. Indeed, parkinsonism is not uncommon in AD. Extrapyramidal signs can be detected in one-third of AD patients during the course of the disease [47], and parkinsonian features are related to neuronal loss in the substantia nigra and putamen [48]. A previous study reported that 3 out of 4 patients with late-onset AD with parkinsonism carried the $A$ allele of rs2298813 [49]. In a case report, 4 AD patients with parkinsonism and psychiatric symptoms were found to have novel mutations in SORL1, with 2 mutations at the VPS10P region, where rs2298813 is located [50]. Our minor allele carriers also had higher scores on the Mayo fluctuation scale, implying a 
link with features of Lewy body dementia. Moreover, the minor allele of rs2298813 has been found to increase the risk of developing dementia in patients with Parkinson's disease [51]. In addition to its involvement in the APP pathway, SORLA also mediates the trophic pathway involving glial cell linederived neurotrophic factor [52], the absence of which could lead to the loss of dopaminergic neurons [53].

\section{Limitations}

There were several limitations of the current study. First, we did not systemically qualify and quantify the motor symptoms in these patients. Therefore, the postulated relationship between atrophy in the putamen and pallidum and pyramidal/extrapyramidal symptoms warrants further study. Second, these patients were followed up for an average of 2 years, so a longer follow-up duration may better confirm the cognitive or motor effects of the volumetric changes in the putamen and pallidum.

\section{Conclusions}

The homozygous major allele of rs2298813 was associated with larger volumes in the right lentiform nucleus, which were associated with cognitive function and clinical progression in AD. Whereas, the smaller volume of right lentiform nucleus in the minor allele carriers was not associated with cognitive function or clinical progression.

\section{List Of Abbreviations}

$A \beta$, amyloid-beta; $A D$, Alzheimer's disease; $A P O E$, apolipoprotein E; APP, amyloid beta precursor protein (APP); $M C l$, mild cognitive impairment; MMSE, Mini-Mental State Examination; MR, magnetic resonance; SORL1, sortilin-related receptor 1; SORLA, sorting-related receptor with type-A repeats; VPS10P, vacuolar protein sorting 10 protein.

\section{Declarations}

\section{Conflicts of interest:}

The authors declare that they have no conflicts of interest related to the subject matter or materials discussed in this article.

\section{Ethics approval and consent to participate}

The study was approved by the institutional review boards of Taipei Veterans General Hospital. Informed consent was obtained from all patients in accordance with our institutional guidelines and the recommendations of the Declaration of Helsinki.

\section{Consent for publication}


Not applicable.

\section{Availability of data and materials}

The datasets used during the current study are available from the corresponding author on reasonable request.

\section{Competing interests}

The authors declare that they have no competing interests.

\section{Funding}

This study was supported by grants from the National Health Research Institutes, Taiwan (NHRI-11A1-CGCO-05-2225-1), the Ministry of Science and Technology, Taiwan (MOST 109-2314-B-075-052-MY2, 1102321-B-001-011-, 110-2321-B-A49A-502-, 110-2634-F-A49 -005 -). Taipei Veterans General Hospital (V110C-057, VGHUST110-G1-5-1, V111C-216) and the Brain Research Center, National Yang Ming Chiao Tung University from the Featured Areas Research Center Program within the framework of the Higher Education Sprout Project by the Ministry of Education (MOE) in Taiwan.

\section{Authors' contributions}

CC wrote the manuscript. JL, CC, YL and WL contributed to the study concept and design. CC, YL, WL, $A C Y$ and JF contributed to analysis and interpretation of data. YL, WL, YK and JF contributed to acquisition of data. WL, YL and JF revised the manuscript. All authors read and approved the final manuscript.

\section{Acknowledgements}

The authors acknowledge all neuropsychological assessors and patients for their cooperation.

\section{References}

1. Hardy J, Selkoe DJ: The amyloid hypothesis of alzheimer's disease: Progress and problems on the road to therapeutics. Science. 2002;297:353-6.

2. Cacace R, Sleegers K, Van Broeckhoven C: Molecular genetics of early-onset alzheimer's disease revisited. Alzheimers Dement. 2016;12:733-48.

3. Rosenberg RN, Lambracht-Washington D, Yu G, Xia W: Genomics of alzheimer disease: A review. JAMA Neurol. 2016;73:867-74.

4. Andersen $\mathrm{OM}$, Rudolph IM, Willnow TE: Risk factor sorl1: From genetic association to functional validation in alzheimer's disease. Acta Neuropathol. 2016;132:653-65.

5. Rogaeva E, Meng Y, Lee JH, Gu Y, Kawarai T, Zou F, et al.: The neuronal sortilin-related receptor sorl1 is genetically associated with alzheimer disease. Nat Genet. 2007;39:168-77. 
6. Lambert JC, Ibrahim-Verbaas CA, Harold D, Naj AC, Sims R, Bellenguez C, et al.: Meta-analysis of 74,046 individuals identifies 11 new susceptibility loci for alzheimer's disease. Nat Genet. 2013;45:1452-8.

7. Miyashita A, Koike A, Jun G, Wang LS, Takahashi S, Matsubara E, et al.: Sorl1 is genetically associated with late-onset alzheimer's disease in japanese, koreans and caucasians. PLoS One. 2013;8:e58618.

8. Reitz C, Cheng R, Rogaeva E, Lee JH, Tokuhiro S, Zou F, et al.: Meta-analysis of the association between variants in sorl1 and alzheimer disease. Arch Neurol. 2011;68:99-106.

9. Scherzer CR, Offe K, Gearing M, Rees HD, Fang G, Heilman CJ, et al.: Loss of apolipoprotein e receptor Ir11 in alzheimer disease. Arch Neurol. 2004;61:1200-5.

10. Andersen OM, Reiche J, Schmidt V, Gotthardt M, Spoelgen R, Behlke J, et al.: Neuronal sorting proteinrelated receptor sorla/Ir11 regulates processing of the amyloid precursor protein. Proc Natl Acad Sci U S A. 2005;102:13461-6.

11. Alexopoulos P, Guo LH, Kratzer M, Westerteicher C, Kurz A, Perneczky R: Impact of sorl1 single nucleotide polymorphisms on alzheimer's disease cerebrospinal fluid markers. Dement Geriatr Cogn Disord. 2011;32:164-70.

12. Chou CT, Liao YC, Lee WJ, Wang SJ, Fuh JL: Sorl1 gene, plasma biomarkers, and the risk of alzheimer's disease for the han chinese population in taiwan. Alzheimers Res Ther. 2016;8:53.

13. K TC, Lunetta KL, Baldwin CT, McKee AC, Guo J, Cupples LA, et al.: Association of distinct variants in sorl1 with cerebrovascular and neurodegenerative changes related to alzheimer disease. Arch Neurol. 2008;65:1640-8.

14. Vardarajan BN, Zhang Y, Lee JH, Cheng R, Bohm C, Ghani M, et al.: Coding mutations in sorl1 and alzheimer disease. Ann Neurol. 2015;77:215-27.

15. Huang CC, Liu ME, Kao HW, Chou KH, Yang AC, Wang YH, et al.: Effect of alzheimer's disease risk variant rs3824968 at sorl1 on regional gray matter volume and age-related interaction in adult lifespan. Sci Rep. 2016;6:23362.

16. Li H, Lv C, Yang C, Wei D, Chen K, Li S, et al.: Sorl1 rs1699102 polymorphism modulates age-related cognitive decline and gray matter volume reduction in non-demented individuals. Eur $\mathrm{J}$ Neurol. 2017;24:187-94.

17. Yin RH, Li J, Tan L, Wang HF, Tan MS, Yu WJ, et al.: Impact of sorl1 genetic variations on mri markers in non-demented elders. Oncotarget. 2016;7:31689-98.

18. Liang Y, Li H, Lv C, Shu N, Chen K, Li X, et al.: Sex moderates the effects of the sorl1 gene rs2070045 polymorphism on cognitive impairment and disruption of the cingulum integrity in healthy elderly. Neuropsychopharmacology. 2015;40:1519-27.

19. McKhann GM, Knopman DS, Chertkow H, Hyman BT, Jack CR, Jr., Kawas CH, et al.: The diagnosis of dementia due to alzheimer's disease: Recommendations from the national institute on agingalzheimer's association workgroups on diagnostic guidelines for alzheimer's disease. Alzheimers Dement. 2011;7:263-9. 
20. Ferman TJ, Smith GE, Boeve BF, Ivnik RJ, Petersen RC, Knopman D, et al.: Dlb fluctuations: Specific features that reliably differentiate dlb from ad and normal aging. Neurology. 2004;62:181-7.

21. Chen CS, Ouyang P, Yeh YC, Lai CL, Liu CK, Yen CF, et al.: Apolipoprotein e polymorphism and behavioral and psychological symptoms of dementia in patients with alzheimer disease. Alzheimer Dis Assoc Disord. 2012;26:135-9.

22. Folstein MF, Folstein SE, McHugh PR: "Mini-mental state". A practical method for grading the cognitive state of patients for the clinician. J Psychiatr Res. 1975;12:189-98.

23. Wechsler D: Wms-iv: Wechsler memory scale 4th edition. New York, NY: The Psychological Corporation. 2009.

24. Vanderploeg RD, Schinka JA, Jones T, Small BJ, Graves AB, Mortimer JA: Elderly norms for the hopkins verbal learning test-revised. Clin Neuropsychol. 2000;14:318-24.

25. Harrison JE, Buxton P, Husain M, Wise R: Short test of semantic and phonological fluency: Normal performance, validity and test-retest reliability. Br J Clin Psychol. 2000;39:181-91.

26. Lu L, Bigler ED: Normative data on trail making test for neurologically normal, chinese-speaking adults. Appl Neuropsychol. 2002;9:219-25.

27. Mack WJ, Freed DM, Williams BW, Henderson VW: Boston naming test: Shortened versions for use in alzheimer's disease. J Gerontol. 1992;47:P154-8.

28. Schmidt C, Wolff M, Weitz M, Bartlau T, Korth C, Zerr I: Rapidly progressive alzheimer disease. Arch Neurol. 2011;68:1124-30.

29. Fischl B, Salat DH, Busa E, Albert M, Dieterich M, Haselgrove $C$, et al.: Whole brain segmentation: Automated labeling of neuroanatomical structures in the human brain. Neuron. 2002;33:341-55.

30. Segonne F, Pacheco J, Fischl B: Geometrically accurate topology-correction of cortical surfaces using nonseparating loops. IEEE Trans Med Imaging. 2007;26:518-29.

31. Bralten J, Arias-Vasquez A, Makkinje R, Veltman JA, Brunner HG, Fernandez G, et al.: Association of the alzheimer's gene sorl1 with hippocampal volume in young, healthy adults. Am J Psychiatry. 2011;168:1083-9.

32. Albin RL, Young AB, Penney JB: The functional anatomy of basal ganglia disorders. Trends Neurosci. 1989;12:366-75.

33. Roh JH, Qiu A, Seo SW, Soon HW, Kim JH, Kim GH, et al.: Volume reduction in subcortical regions according to severity of alzheimer's disease. J Neurol. 2011;258:1013-20.

34. Pini L, Pievani M, Bocchetta M, Altomare D, Bosco P, Cavedo E, et al.: Brain atrophy in alzheimer's disease and aging. Ageing Res Rev. 2016;30:25-48.

35. de Jong LW, van der Hiele K, Veer IM, Houwing JJ, Westendorp RG, Bollen EL, et al.: Strongly reduced volumes of putamen and thalamus in alzheimer's disease: An mri study. Brain. 2008;131:3277-85.

36. Eustache P, Nemmi F, Saint-Aubert L, Pariente J, Peran P: Multimodal magnetic resonance imaging in alzheimer's disease patients at prodromal stage. J Alzheimers Dis. 2016;50:1035-50. 
37. Tang X, Holland D, Dale AM, Younes L, Miller MI, Alzheimer's Disease Neuroimaging I: Shape abnormalities of subcortical and ventricular structures in mild cognitive impairment and alzheimer's disease: Detecting, quantifying, and predicting. Hum Brain Mapp. 2014;35:3701-25.

38. Cho H, Kim JH, Kim C, Ye BS, Kim HJ, Yoon CW, et al.: Shape changes of the basal ganglia and thalamus in alzheimer's disease: A three-year longitudinal study. J Alzheimers Dis. 2014;40:285-95.

39. Li YD, He HJ, Dong HB, Feng XY, Xie GM, Zhang LJ: Discriminative analysis of early-stage alzheimer's disease and normal aging with automatic segmentation technique in subcortical gray matter structures: A multicenter in vivo mri volumetric and dti study. Acta Radiol. 2013;54:1191-200.

40. Wang ML, Wei XE, Fu JL, Li W, Yu MM, Li PY, et al.: Subcortical nuclei in alzheimer's disease: A volumetric and diffusion kurtosis imaging study. Acta Radiol. 2018;59:1365-71.

41. Braak H, Braak E: Alzheimer's disease: Striatal amyloid deposits and neurofibrillary changes. J Neuropathol Exp Neurol. 1990;49:215-24.

42. Cogswell PM, Wiste HJ, Senjem ML, Gunter JL, Weigand SD, Schwarz CG, et al.: Associations of quantitative susceptibility mapping with alzheimer's disease clinical and imaging markers. Neuroimage. 2021;224:117433.

43. Bartzokis G, Sultzer D, Cummings J, Holt LE, Hance DB, Henderson VW, et al.: In vivo evaluation of brain iron in alzheimer disease using magnetic resonance imaging. Arch Gen Psychiatry. 2000;57:4753.

44. Lee PS, Crammond DJ, Richardson RM: Deep brain stimulation of the subthalamic nucleus and globus pallidus for parkinson's disease. Prog Neurol Surg. 2018;33:207-21.

45. Qi Y, Xu M, Wang W, Wang YY, Liu JJ, Ren HX, et al.: Early prediction of putamen imaging features in hiv-associated neurocognitive impairment syndrome. BMC Neurol. 2021;21:106.

46. Baez S, Pinasco C, Roca M, Ferrari J, Couto B, Garcia-Cordero I, et al.: Brain structural correlates of executive and social cognition profiles in behavioral variant frontotemporal dementia and elderly bipolar disorder. Neuropsychologia. 2019;126:159-69.

47. Scarmeas N, Hadjigeorgiou GM, Papadimitriou A, Dubois B, Sarazin M, Brandt J, et al.: Motor signs during the course of alzheimer disease. Neurology. 2004;63:975-82.

48. Horvath J, Burkhard PR, Herrmann FR, Bouras C, Kovari E: Neuropathology of parkinsonism in patients with pure alzheimer's disease. J Alzheimers Dis. 2014;39:115-20.

49. Cuccaro ML, Carney RM, Zhang Y, Bohm C, Kunkle BW, Vardarajan BN, et al.: Sorl1 mutations in earlyand late-onset alzheimer disease. Neurol Genet. 2016;2:e116.

50. Qiu G, Xu C, Guo Q, Zhu FQ: Sorl1 mutations are associated with parkinsonian and psychiatric features in alzheimer disease: Case reports. Medicine (Baltimore). 2021;100:e25585.

51. Maple-Grodem J, Chung J, Lunde KA, Tzoulis C, Tysnes OB, Pedersen KF, et al.: Alzheimer disease associated variants in sorl1 accelerate dementia development in parkinson disease. Neurosci Lett. 2018;674:123-6. 
52. Glerup S, Lume M, Olsen D, Nyengaard JR, Vaegter CB, Gustafsen C, et al.: Sorla controls neurotrophic activity by sorting of gdnf and its receptors gfralpha1 and ret. Cell Rep. 2013;3:186-99.

53. Lin LF, Doherty DH, Lile JD, Bektesh S, Collins F: Gdnf: A glial cell line-derived neurotrophic factor for midbrain dopaminergic neurons. Science. 1993;260:1130-2.

\section{Figures}

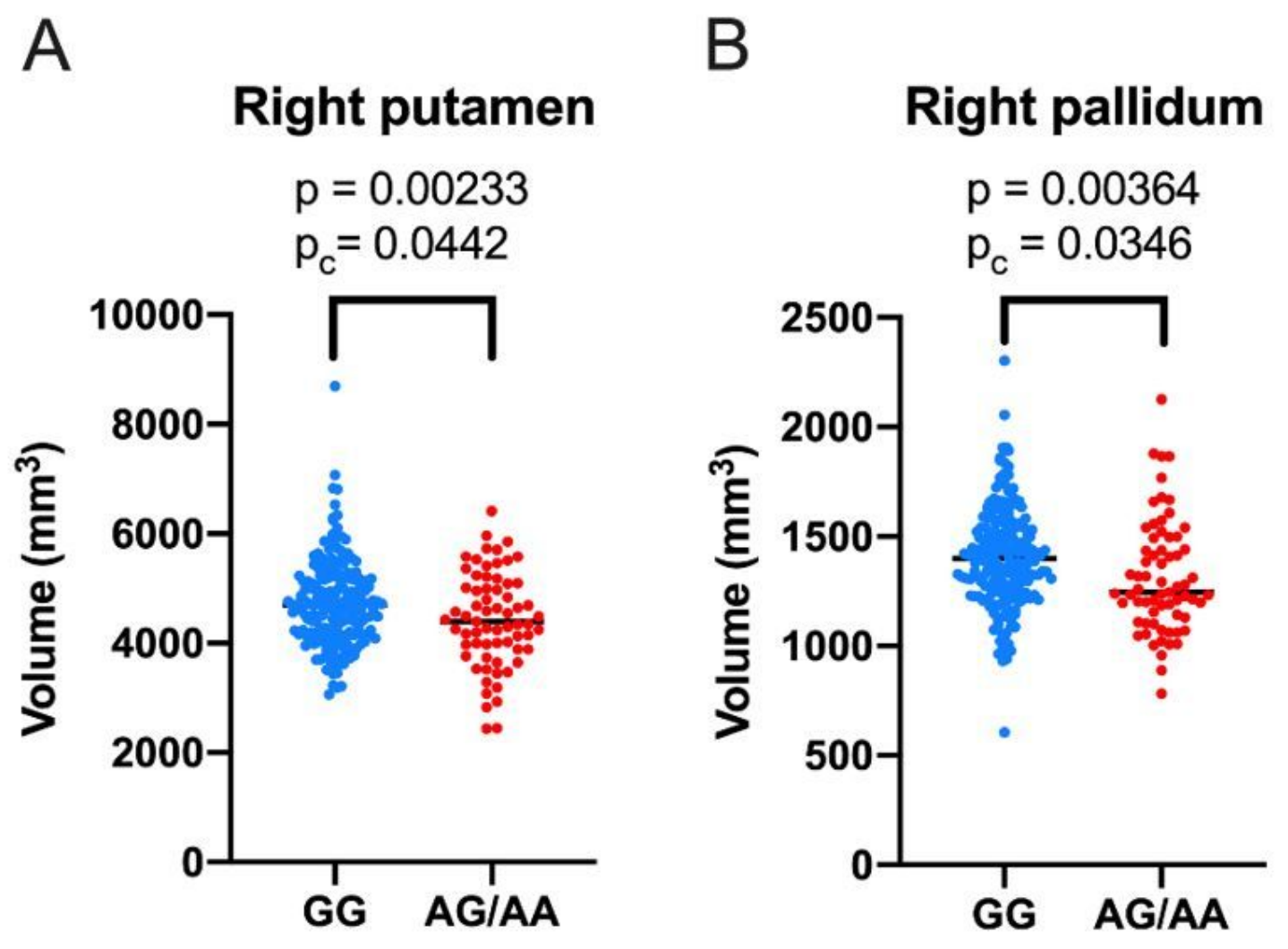

Figure 1

Volumetric comparison of the right putamen $(A)$ and right pallidum (B) between the with and without the minor allele of rs2298813. 